\title{
Analyse des contraintes et degrés de liberté de l'enseignant de mathématiques en formation initiale des professeurs
}

\section{Alain Bronner}

\section{CpenEdition}

Journals

Édition électronique

URL : http://journals.openedition.org/trema/1617

DOI : 10.4000/trema.1617

ISSN : 2107-0997

Éditeur

Faculté d'Éducation de l'université de Montpellier

Édition imprimée

Date de publication : 1 décembre 2000

Pagination : 39-4739-48

ISSN : 1167-315X

Référence électronique

Alain Bronner, «Analyse des contraintes et degrés de liberté de l'enseignant de mathématiques en formation initiale des professeurs », Tréma [En ligne], 18 | 2000, mis en ligne le 23 janvier 2013, consulté le 12 juin 2020. URL : http://journals.openedition.org/trema/1617 ; DOI : https://doi.org/ 10.4000/trema.1617

Ce document a été généré automatiquement le 12 juin 2020.

Trema 


\title{
Analyse des contraintes et degrés de liberté de l'enseignant de mathématiques en formation initiale des professeurs
}

\author{
Alain Bronner
}

\section{Introduction}

1 Ce travail a pour objectif de présenter un dispositif de formation à l'intention des professeurs stagiaires (PLC2) de mathématiques qui a été réalisé à l'IUFM de Montpellier sur la transposition didactique des nombres réels et la racine carrée. Il s'inspire d'une recherche (1997a et 1997b) concernant les rapports personnels et institutionnels des professeurs à ces objets de savoir. Il s'agit, à travers ce dispositif, de provoquer chez les PLC2 une prise de conscience de leur rapport aux objets de savoir «nombre réel» et "racine carrée» et de les faire travailler sur la notion de transposition didactique. Plus généralement, ce dispositif a aussi comme ambition de dégager des types de contraintes qui pèsent sur l'enseignant et faire émerger les degrés de liberté des sujets de l'action didactique dans l'institution de l'enseignement secondaire.

\section{L'étude sur les nombres réels et la racine carrée}

Dans des travaux antérieurs (1997 et 1998), j'ai mené une étude sur l'enseignant dans le cadre de l'approche anthropologique développée par Y. Chevallard (1988). Du point de vue méthodologique, j'ai réalisé, d'une part des questionnaires, et d'autre part des entretiens semi-directifs en m'appuyant sur un guide d'entretien (annexe). Je commencerai par présenter l'étude du rapport personnel d'un enseignant, que j'appelle Pierre $^{1}$, dont certains éléments, apparus avec force, tout au long de l'interview ont 
particulièrement attiré mon attention. J'étudierai ensuite le rapport de l'institution aux objets "nombre réel» et "racine carrée ». Je poursuivrai en exposant l'analyse des différents types de rapports d'enseignants rencontrés.

\section{Une position de conformité stricte : le rapport personnel de Pierre}

3 Pierre a un peu moins de quarante ans, enseigne les mathématiques dans un collège d'une petite ville et a des classes de Troisième depuis plusieurs années. J'ai pu constater que la position de Pierre se caractérise tout d'abord par l'importance qu'il accorde à la maitrise des savoir-faire basés sur les règles algébriques (comme celles qui sont liées à la racine carrée d'un produit par exemple). En effet, les manipulations formelles constituent les objectifs prioritaires de Pierre. L'allusion à ce type de travail formel constitue un thème récurrent dans le discours de ce professeur tout au long de l'entretien : "La racine carrée, on est obligé de passer pas mal de temps sur la technique ». On peut, à travers son discours, voir émerger un objet didactique «[...] la technique »(de la racine carrée), qui occupe une place importante dans son enseignement. Sans doute, la présence des règles algébriques concernant l'objet "racine carrée " dans les programmes officiels ${ }^{2}$ (depuis l'arrêté du 14 novembre 1985) et leur mise en relief dans la «colonne» des compétences exigibles constituent une contrainte forte pour les enseignants. Ainsi, en décrivant le début de sa «leçon» sur la racine carrée, Pierre indique qu'il présente quelques exemples de carrés et de racines carrées, et ajoute: "Après ce travail-là je donne la définition et la notation. Après je fais un travail plus fastidieux pendant plusieurs séances sur la technique». Ce discours nous fait bien ressentir cette préoccupation constante pour « la technique».

4 On sait que la notion de racine carrée a joué un rôle fondamental dans l'histoire et la culture mathématique des nombres réels. Elle est même associée à des obstacles à la connaissance mathématique, qui ont laissé des traces comme l'atteste l'expression «crise des irrationnels» à propos de la découverte de l'incommensurabilité de grandeurs comme la diagonale d'un carré, le côté étant pris comme unité. L'enseignement de la racine carrée, proposé par Pierre, n'est pas lié à une ouverture vers les irrationnels, ou une perspective d'approche des nombres réels : « Non, on ne leur donne pas. Je ne l'ai pas vu dans les programmes et je ne le fais pas ".

5 Ainsi, à partir des règles algébriques, la technique de la racine carrée prend une importance particulière dans l'entretien de Pierre. Les raisons avancées par les enseignants, qui se situent dans une position identique à Pierre, évoquent en général les contraintes institutionnelles plutôt que l'objet de savoir culturel: «c'est le programme »disent certains, tandis que d'autres expriment très fort le projet de faire réussir les élèves au Brevet. D'ailleurs le professeur veut apparaitre comme un «bon sujet» de l'institution et il approuve les choix des programmes concernant les connaissances sur les nombres et l'irrationalité : "Je trouve que c'est un bien. Les élèves ne voyaient absolument pas pourquoi ». Il y va même d'une critique de l'enseignement du Numérique dans les périodes précédentes: "Il y a quelques années, on introduisait les irrationnels, je ne me souviens plus très bien comment on faisait. [...] Cela passait bien pour un quart de la classe, mais je me demande s'ils comprenaient bien... ». 
6 En fait le rapport de Pierre à l'objet racine carrée se trouve bien en conformité avec le rapport de l'institution EMS (enseignement mathématique secondaire). Comme pour Pierre, chez de nombreux enseignants de notre étude, on note une absence d'aspect «nouveau nombre » dans leur enseignement et l'on ne décèle pas d'enjeux didactiques liés à l'aspect "nouveau nombre", tandis que certains apprentissages algébriques envahissent l'espace didactique à travers l'objet dit de «la technique de la racine carrée». Je dirai alors que ces enseignants sont dans une position de "conformité stricte» relativement au rapport de l'institution EMS dont je rappelle maintenant quels traits significatifs dans la période actuelle.

\section{Le rapport de l'institution EMS aux objets « nombre réel » et « racine carrée »}

7 J'emploierai le terme rapport de EMS, au sens du rapport institutionnel, qui peut être «lu » à partir de tout ce que l'institution donne à voir (programmes ${ }^{3}$, instructions, notes... ). Les transformations d'écritures des nombres font entrer l'apprentissage du Numérique dans une perspective essentiellement algébrique dissociée de la mesure des grandeurs. De plus, on ne trouve pas explicitement, que ce soit pour certains quotients de nombres décimaux, ou pour certaines racines carrées, une référence à un aspect " nouveau nombre ", c'est-à-dire une référence à un caractère d'irrationalité ou au fait que certains des nombres obtenus ne sont pas des décimaux. Je rappelle que cela n'a pas toujours été le cas dans cette institution, et notamment à la période des mathématiques modernes. J'ai proposé (Bronner, 1997a) d'appeler, le caractère de nondécimalité, par un nom propre, l'idécimalité, compte tenu de l'importance jouée par les nombres décimaux dans la construction du Numérique.

8 En Seconde, au lycée, la perspective algébrique est poursuivie et l'ensemble Rapparaît comme "un grand fourre-tout» qui s'élabore sur une notion pré-construite de "nombre », indépendant de sa nature arithmétique, et où les règles de calcul vont être officialisées une fois pour toutes, la nature des nombres étant mise à l'écart. La seule précision sur les réels se cristallise dans la remarque suivante : «S'ajoutent en -Seconde [...] les notations $N, Z, Q, R$; sur ces différents points, il s'agit d'un simple vocabulaire et aucun développement n'est au programme "(arrêté du 25 avril 1990).

9 L'institution EMS conduit actuellement l'objet «nombre réel » à une crise d'identité. L'analyse de l'enseignement du Numérique de 1850 à nos jours m'a amené à proposer la notion de vide institutionnel (Bronner, 1997a) à propos de la négociation du passage des décimaux ou des rationnels aux réels.

\section{Principaux résultats de l'étude des rapports des enseignants à l'objet « nombre réel »}

Cette étude est centrée autour des questions suivantes: Comment le vide institutionnel est-il perçu par les enseignants? Comment le traitent-ils ? La plupart des rapports des enseignants aux objets «nombre réel » sont conformes au rapport de l'institution EMS, mais sans nécessairement présenter une conformité stricte comme pour le professeur Pierre. En fait, l'étude (à partir des questionnaires et entretiens) fait tout d'abord apparaître l'importante diversité des rapports des enseignants de 
Troisième à l'objet «nombre réel ». Ce résultat m'a conduit à proposer la configuration suivante de positions d'enseignants de Troisième quant à l'objet nombre réel sur la population étudiée :

- position de conformité stricte (comme celle de Pierre);

- position d'ouverture vers les «nouveaux nombres »;

- position d'ouverture vers les idécimaux ;

- position d'ouverture assumée vers les irrationnels.

\section{Une position d'ouverture vers les « nouveaux nombres »}

11 Tout en prenant en compte les aspects algébriques de l'enseignement des quotients et des racines carrées comme dans la position de conformité stricte, certains enseignants s'inscrivent dans une position où ils placent la séquence sur la racine carrée dans une ouverture vers des «nouveaux nombres ». Ces professeurs souhaitent faire comprendre aux élèves que certaines fractions et racines carrées, non décimales, ont un statut de nombre; cependant ils ne parlent pas d'irrationalité, ni d'idécimalité, à propos des racines carrées. De plus ils introduisent une nouvelle catégorie de nombres: des «nouveaux nombres", disent-ils; ou encore ils parlent de "valeur exacte». Un tel rapport d'enseignant, repéré chez Patrick, sera ainsi nommé: une position d'ouverture vers les " nouveaux nombres".

Chez Patrick, le statut de nombre des rationnels et des racines carrées constitue l'enjeu didactique fondamental, qu'il exprime de façon récurrente dans son discours : «savoir que est l'écriture d'un nombre en valeur exacte qui peut être approché ensuite et qu'on ne sait pas l'écrire autrement. Moi, j'aimerais arriver à faire comprendre qu'en fait c'est un nombre qu'on ne sait pas écrire, qu'il a fallu inventer un symbole pour ça, un peu comme $\pi$. ». Ainsi une obsession intense à faire voir, dans le discours, les racines carrées et les fractions sous un «aspect nombre" se fait jour. Malgré cela, les aspects de véritable «nouveau nombre » à caractère irrationnel ou idécimal, ne sont pas explicités dans le discours recueilli. Cette position d'ouverture vers les nouveaux nombres, observée chez Patrick, représente pour moi une tentative de réduction du vide didactique institutionnel sur les réels, qui maintient, quand même, une grande conformité avec le rapport au Numérique de EMS.

\section{Position d'ouverture vers les idécimaux}

Certains enseignants s'imposent d'introduire un travail sur la nature idécimale de certaines racines carrées comme $\sqrt{2}, \sqrt{ } 7$. Ils s'inscrivent dans le prolongement de la position précédente d'ouverture vers des "nouveaux nombres", et vont essayer d'expliciter cet aspect, ou encore de le problématiser à l'aide d'une notion d'idécimalité. L'opposition "décimal-idécimal» est alors assumée. Ils peuvent se limiter à traduire le phénomène par l'expression des «nombres qui ne tombent pas justes ». Mais ils peuvent aussi proposer des exercices pour montrer que ces nombres ne sont pas des décimaux, sans toutefois démontrer que ce sont des irrationnels. Je nomme alors un tel rapport aux objets d'enseignement «nombre réel» et « racine carrée » une position d'ouverture vers les idécimaux. Ce rapport institutionnel d'enseignant comme je l'ai repéré chez Claude est encore une position où se négocie 
une réduction du vide didactique sur les nombres réels, en jouant sur l'opposition décimale-idécimale.

Claude rend public des éléments de son rapport personnel à ces nombres à propos d'une question sur les difficultés d'apprentissage : «Ils le comprennent, mais difficilement; on est obligé de leur faire admettre que ces racines-là ce ne sont pas des nombres normaux, ce sont des nombres qui ne tombent jamais justes ».Ainsi, pour Claude, les nombres « normaux ", les nombres qui "permettent d'écrire les résultats", les nombres qui «tombent juste ", les nombres que l'on peut écrire en "totalité », ces nombres sont bien sûr les décimaux. Cette position d'ouverture vers les idécimaux peut permettre de problématiser et de donner du sens à l'artefact didactique de "valeur exacte », et plus généralement de combler une partie du vide didactique.

\section{Position assumée d'ouverture vers les irrationnels}

Parmi les enseignants ayant participé aux questionnaires ou interviews, quelques-uns envisagent de présenter explicitement une notion de nombre irrationnel à travers l'enseignement de la racine carrée. Certains introduisent même des connaissances permettant un traitement de l'irrationalité. Pour comprendre les raisons de cette position, on peut avancer le poids culturel de l'irrationalité des racines carrées (Assude, 1991). Mais il peut aussi y avoir une nécessité didactique de montrer la nature de «nouveau nombre », nécessité qui peut être interprétée comme une forme de réponse au vide didactique. Cette position permet de traiter l'artefact didactique de «valeur exacte ", comme avec la position précédente d'ouverture vers les idécimaux, mais en se situant davantage dans la perspective culturelle de l'opposition rationnelleirrationnelle. Je la nomme position d'ouverture vers les irrationnels.

16 J'ai pu aussi montrer (Bronner, 1997a) que les rapports des enseignants de Seconde ne présentent pas de différences significatives par rapport à ceux de Troisième, et s'inscrivent dans l'espace des éventualités repérées pour la classe de Troisième. Je fais l'hypothèse que ces phénomènes - la variété des rapports d'enseignants et l'absence de différence entre les deux classes de Troisième et de Seconde - sont dus à la présence du vide institutionnel qui laisse une importante ouverture pour le savoir enseigné.

\section{Le dispositif de formation à l'intention des Professeurs stagiaires (PLC2)}

17 Le travail a commencé à l'IUFM en répartissant les professeurs stagiaires PLC2 par binômes et en essayant de maintenir la complémentarité professeur de collège professeur de seconde, dans la mesure des possibilités ${ }^{4}$. Les PLC2 devaient ensuite s'interviewer mutuellement à l'aide du guide d'entretien (annexe) qui leur a été fourni. Cette première phase avait comme objectif de faire émerger quelques éléments de leur rapport personnel aux objets «nombre réel » et « racine carrée », et de s'approprier le but du travail et le guide d'entretien. L'analyse précédente du rapport de l'institution EMS a alors été exposée et a débouché sur une première rencontre avec le concept de transposition didactique.

Dans une deuxième phase, les PLC2 sont allés interviewer leur conseiller pédagogique tuteur ${ }^{5}$ ainsi qu'un autre professeur de leur établissement avec le guide d'entretien. 
Une phase de restitution à l'IUFM était prévue ensuite pour souligner les principales caractéristiques des réponses des enseignants par rapport aux questions de la recherche. Elle a permis de mettre en évidence la grande variabilité de leurs réponses sur la question de l'enseignement des nombres et de la racine carrée et de s'interroger sur l'importance de cette variabilité.

Les résultats de la recherche ont été présentés en les reliant aux observations et aux analyses des PLC2. En retour, ces outils ont permis de retravailler le matériel apporté par les PLC2.

Il s'est avéré que les conseillers pédagogiques tuteurs répondent volontiers aux questions portant sur leurs pratiques didactiques et pédagogiques. Par contre, la partie du questionnaire mettant en évidence leur rapport personnel au savoir, qui pourrait dévoiler une certaine composante privée, est éludée. Cette constatation suggère, pour la poursuite et pour la reprise du dispositif, de placer ces enseignants dans leur position institutionnelle de tuteurs, en proposant aux PLC2 de remplir eux-mêmes le questionnaire et de demander à leurs tuteurs d'en discuter, ce qui serait une façon, à travers les contributions recueillies de pouvoir mieux saisir ce rapport personnel aux objets de savoir en jeu.

\section{Conclusion}

Ce dispositif a permis de travailler la transposition des nombres et de la racine carrée avec les PLC2, et d'introduire le concept de transposition didactique (Chevallard, 1985).

On a mis en évidence, à propos d'un objet comme celui de "nombre réel », qu'il y a plusieurs façons d'être enseignant de Troisième: il y a plusieurs positions d'enseignants relativement à ces objets. Les rapports institutionnels des enseignants sont partiellement déterminés par les contraintes institutionnelles, et principalement par le rapport au Numérique de EMS, mais qu'une grande place reste offerte pour la diversité en lien avec des préoccupations didactiques et l'épistémologie spontanée de chacun. Cette grande ouverture de l'espace des positions des enseignants est peut-être amplifiée ici par le vide didactique institutionnel relativement à l'objet " nombre réel », objet qui reste éminemment problématique pour les institutions d'enseignement depuis de nombreuses périodes (Bronner, 1997a).

On a pu souligner aussi les effets de naturalisation (Cheval-lard, 1996) du rapport institutionnel à des objets d'enseignement. Des conflits entre différentes dimensions du rapport personnel à l'objet «nombre réel » de certains enseignants ont pu émerger. Par exemple, au-delà de la conformité stricte dont le discours de Pierre témoigne, l'analyse de l'entretien révèle que le rapport personnel de Pierre au Numérique présente des éléments qui sont profondément en conflit. Tout d'abord, dans ce discours, on peut percevoir les effets d'assujettissements anciens à d'autres institutions que EMS (université, l'institution EMS de périodes antérieures...) :

"J'étais toujours fasciné pendant mes études par cette façon d'agrandir à chaque fois les ensembles de nombres et d'arriver à trouver les propriétés qui se prolongent. Mais on a du mal à abandonner un certain nombre de choses et on a été formé comme cela. [...] Mais c'est vrai qu'au début c'est comme cela qu'on présentait les ensembles de nombres. Maintenant la preuve c'est que je n'y pensais plus. En plus de cela, avec les difficultés que rencontrent les gamins, je crois qu'au fur et à mesure on élague ». 
Ce conflit interne issu d'une forme d'incompatibilité de la composante institutionnelle avec d'autres dimensions du rapport personnel de Pierre, tend à montrer que, pour certains enseignants, au bout d'un certain temps, les programmes sont intériorisés comme s'ils avaient toujours été déjà là. Cela risque encore d'être plus prégnant chez les PLC2 qui n'ont rencontré ces programmes que lors de leur entrée en fonction comme enseignant. Ce travail peut ainsi les mettre en alerte sur le caractère, certes officiel, mais daté, conventionnel et très relatif des programmes qu'ils ont à enseigner.

\section{BIBLIOGRAPHIE}

ASSUDE T. : Un phénomène, d'arrêt de la transposition didactique. Écologie de l'objet « Racine, carrée » et analyse du curriculum. Thèse : Université de Grenoble, 1992.

BRONNER A. : Étude didactique des nombres réels, idécimalité et racine carrée. Thèse : Université J. Fourier, Grenoble, 1997a.

BRONNER A. : « Les rapports d'enseignants de Troisième et de Seconde aux objets nombre réel et racine carrée ", in Recherches en Didactique des Mathématiques, Vol. 17, № 3. Grenoble, La pensée sauvage, $1997 \mathrm{~b}$.

CHEVALLARD Y. : La transposition didactique. Grenoble, La pensée sauvage, 1985.

CHEVALLARD Y : « Le concept de rapport au savoir », in Actes de Séminaire de didactique des Mathématiques et de l'informatique. Grenoble, Université J. Fourier, 1988-89.

CHEVALLARD Y. :» La fonction professorale : Esquisse d'un modèle didactique », in Actes de la VIII École d'été de didactique des mathématiques. Clermont-Ferrand, IREM de Clermont-Ferrand, 1996.

\section{ANNEXES}

\section{Annexe}

\section{Le guide d'entretien ${ }^{6}$}

1) Selon vous, à quel niveau (classe) les élèves sont-ils confrontés aux nombres réels, et en particulier aux irrationnels? Et dans quels contextes?

Dans votre enseignement, quelles difficultés rencontrez-vous qui vous semblent avoir un rapport avec les connaissances des élèves sur les nombres réels?

2) Selon vous, quelles connaissances ont les élèves actuels de fin de Troisième sur la notion de nombre réel? 
3) Selon vous, à quel niveau (classe) pensez-vous que la notion de nombre décimal est acquise pour la majorité des élèves? Qu'est-ce qui est effectivement acquis (propriétés, sens, etc.) ? Même question pour la notion de « rationnel».

4) Selon vous, comment les élèves en fin de Troisième, actuellement, différencient-ils les décimaux, les rationnels (non décimaux) et les irrationnels?

5) Quels sont vos objectifs (connaissances, savoir-faire... ) à propos de l'apprentissage de ces nombres (réels, décimaux, rationnels, irrationnels) en fin de Troisième?

6) Que représente pour vous la notion de racine carrée sur le plan mathématique ?

7) Quelles situations ou quels cadres proposez-vous pour introduire la notion de racine carrée ? Quelles difficultés ou quelles erreurs fait-elle surgir? Quelles sont, selon vous, les origines possibles de ces difficultés ou erreurs?

8) Selon vous, quelles connaissances ont les élèves sur les nombres du type $\sqrt{ } 17$ et quelles significations en donnent-ils en fin de Troisième ? Proposez-vous parfois la résolution d'équations du type $x^{2}=\sqrt{17}$ ? Pourquoi?

9) De nombreux travaux sur la notion de racine carrée montrent que, pour certains élèves de fin de Troisième, l'équation $\mathrm{x}^{2}=17 \mathrm{n}$ 'a pas de solution, ou alors a pour solution un décimal qui est une valeur approchée de $\sqrt{17}$. Qu'en pensez-vous?

10) Attachez-vous une grande importance dans votre enseignement à différencier " valeur exacte » et " valeur approchée »? Pourquoi ? Comment expliquez-vous à vos élèves la différence entre valeur exacte et valeur approchée de $V 17$ ? En particulier, que dites-vous sur la « valeur exacte de $\sqrt{ } 17 »$ ?

11) Utilisez-vous la calculatrice dans la séquence sur la notion de racine carrée ? Dans quels buts? Est-elle source de difficultés ? Si oui, lesquelles?

12) Comment introduisez-vous et définissez-vous la fonction racine carrée aux élèves ? En Seconde, quels objectifs vous fixez-vous à propos de la fonction racine carrée?

13) Quels problèmes donnez-vous à vos élèves de Seconde utilisant la représentation graphique de la fonction « racine carrée » ? Dans quels buts ?

14) Posez-vous des questions du type « quelle est l'image par la fonction racine carrée du nombre $\sqrt{ } 17 »$ ? Pourquoi?

\section{NOTES}

1. J'ai choisi de désigner les enseignants par des prénoms masculins ou mixtes. Je précise, à propos des enseignants interviewés, qu'ils ont au minimum quinze années d'exercice.

2. Je fais référence ici aux programmes et instructions officielles en vigueur au moment des interviews et questionnaires.

3. Il s'agit des programmes de 1985 à 1995 pour le collège.

4. Une moitié (environ) des professeurs a en charge une classe de Seconde et l'autre moitié une classe de collège.

5. Pendant leur année de stage les professeurs stagiaires sont encadrés par un professeur : le conseiller pédagogique tuteur.

6. Les questions 12,13 et 14 (Cf.annexe) ont été adaptées pour les professeurs de Troisième. 


\section{RÉSUMÉS}

Cet article présente un dispositif réalisé à l'IUFM de Montpellier sur la transposition didactique des nombres réels et de la racine carrée à l'intention des professeurs stagiaires PLC2 de mathématiques. Il s'agissait notamment, à travers ce dispositif, de provoquer chez les PLC2 une prise de conscience de leur rapport aux objets de savoir «nombre réel » et « racine carrée ". Enfin, ce travail a permis de dégager des types de contraintes qui pèsent sur l'enseignant dans l'institution de l'enseignement secondaire.

This article presents a scheme realised at the "IUFM de Montpellier" teacher training college on the didactic transposition of "Real" and "Square Root" numbers for the benefit of second year IUFM mathematics students. The point was, specifically speaking, by means of this scheme, to induce in these second year IUFM students, an awareness of their relationship to the intellectual objects: "Real" and "Square Root" numbers. Lastly, this research work made it possible to reveal the various types of constraints which hem in the teacher within the institution that is secondary school.

\section{INDEX}

Keywords : didactic transposition, educational institution, real number, relationship to an intellectual object, square root

Mots-clés : institution, nombre réel, racine carrée, rapport à un objet de savoir, transposition didactique

\section{AUTEUR}

\section{ALAIN BRONNER}

IUFM de Montpellier, équipe ERES, Université Montpellier II 